\title{
MEASUREMENT OF INFRARED TRANSITION SPECTRUM OF SYNCHROTRON RADIATION THROUGH SUPERCONDUCTING $\mathrm{YBa}_{2} \mathrm{Cu}_{3} \mathrm{O}_{7-\delta}$ FILM
}

\author{
S.I. Tyutyunnikov, V.N. Shaliapin
}

Joint Institute for Nuclear Research, Moscow Region, 14980 Dubna, Russian Federation

\author{
AND N. SCINTEE \\ Institute for Atomic Physics, Bucharest, Romania
}

\begin{abstract}
This paper presents measurement results of the synchrotron radiation infrared transmission spectrum through YBCO film on $\mathrm{MgO}$ substrate at $T=20 \mathrm{~K}$ temperature. The film's transmission temperature of the superconducting state is $T_{\mathrm{c}}=80 \mathrm{~K}$. During formation cycle the maximum intensity wavelength varies from $100 \mu \mathrm{m}$ to $10 \mu \mathrm{m}$. Due to spectrum evolution the detector signal that gives synchrotron radiation sample transmission is related to the sample transmission ability, the sensitivity of the detector and synchrotron radiation spectrum via an integral equation. The best agreement with experiment results is achieved for the $E \gamma=20 \mathrm{meV}$ energy gap value, which corresponds to $2 E \gamma / k T_{\mathrm{c}}=2.9$ at $T=20 \mathrm{~K}$.
\end{abstract}

PACS numbers: $78.30 .-\mathrm{j}, 74.76 .+\mathrm{w}$

Middle and far range infrared (IR) spectroscopy have achieved good progress. There are used Fourier interferometers and black body radiation sources of $T=1000 \div 1500 \mathrm{~K}$ temperatures with $p=10^{-8} \mathrm{~W}$ radiation power in the $\Delta \lambda / \lambda=0.1$ region. A further increase in spectroscopic investigation efficiency depends on highly intensive radiation sources development in the $\lambda \geq 100 \mu \mathrm{m}$ range.

These sources can be cyclic electron accelerators (synchrotron radiation sources (SR) and variable magnetic structure sources (free electron lasers - FEL) [1].

The difference between SR sources and a black body source in the far IR range is that the spectral density of the former decreases as $\lambda^{-1}$ while for the latter as $\lambda^{-3}$ [2].

SR-source radiation flux was measured in the long wave region at the Brookhaven NSLS source [1]. The results showed that in the spectral range $\lambda>100 \mu \mathrm{m}$ the power ratio of the synchrotron radiation to the black body radiation is $P_{\mathrm{SL}} / P_{\mathrm{b} . \mathrm{b} .}=10[3]$. 
In specialized storage systems the maximum power is peaked in the soft X-ray range and only a small part is in infrared range. SR spectrum is characterized by a parameter $\lambda_{c}=4.2 R / \gamma^{3}$, where $R$ is the electron ring radius and $\gamma$ the relativistic factor.

For the NSLS storage ring one has $R=2.2 \mathrm{~m}$ and $\lambda_{c}=59 \AA$ [3]. To estimate the radiation power in the far IR range one uses the following asymptotic formula $\left(\lambda \gg \lambda_{c}\right)[1]$ :

$$
N_{\lambda} \approx 5.27 \times 10^{16} \times I_{\mathrm{e}}[\mathrm{A}] E[\mathrm{GeV}]\left(\frac{\lambda_{c}}{\lambda}\right)^{1 / 3} \frac{\Delta \lambda}{\lambda},
$$

where $I_{\mathrm{e}}$ is the electron ring current in amperes and $E$ is the energy in $\mathrm{GeV}$. Higher SR power in the infrared range is possible if one has:

- larger horizontal and vertical apertures of the optical channel;

- a higher current accumulated in the ring.

The available maximum values of these parameters at the NSLS [3] are

$$
e_{X}=90 \mathrm{mrad}, e_{Z}=90 \mathrm{mrad}, I_{\mathrm{e}}=750 \mathrm{~mA} \text {. }
$$

Substituting $I_{\mathrm{e}}=N_{\mathrm{e}} e c /(2 \pi R)$ and $\lambda_{\mathrm{c}}=R / E^{3}$ in Eq. (1) we get $N(\lambda) \approx N_{\mathrm{e}} / R^{2 / 3}$.

This shows that the best radiator for the IR range is an electron ring with a minimum radius $R$ and a maximum number of electrons. as is shown in Ref. [4] a pulsed electron accumulator is the most suitable for one SR generation in the submillimetric range. The parameters of the electron ring for the pulsed accumulator are

- $R=3 \div 4 \mathrm{~cm}$,

- $E_{\mathrm{e}}=20 \div 25 \mathrm{MeV}$,

- $N_{\mathrm{e}}=5 \times 10^{12}$,

- $I_{\mathrm{e}}=1200 \div 1300 \AA[5]$.

The absolute power was also measured at $T=4.2 \mathrm{~K}$ by an InSb detector with a set of narrow-band interface filters. The radiation power - measured for the vertical acceptance $\theta_{z}=30 \mathrm{mrad}, \lambda=300 \mu \mathrm{m}$ and $\Delta \lambda / \lambda=0.1-$ was $P(\lambda)=$ $1.5 \times 10^{-5} \mathrm{~W}$. This corresponds to $5 \times 10^{-4} \mathrm{~W} / \mu \mathrm{m}$ in terms of total ring radiation power.

The calculated value of $P$ is $P(\lambda)=6 \times 10^{-4} \mathrm{~W} / \mu \mathrm{m}$ for $N_{\mathrm{e}}=2 \times 10^{12}$. The measurement error was $30 \%$. The linear relation between radiation power and the number of electrons, for any number of electrons in its variation range, is observed.

High temperature superconductor (HTSC) materials were studied for effects that manifest as a smooth dependence of transmission or reflection on the wavelength.

The fact that during electron ring formation in the pulsed accumulator the short-wave boundary of the SR spectrum moves towards shorter waves and time evolution of the spectrum takes place can be employed.

The object of interest is placed in front of the detector (Fig. 1). We find that the transmission factor of the object $T(\lambda)$ is connected with the time function of the detector signal $U(t)$ through an integral equation

$$
U(t)=\int_{\lambda 1}^{\lambda 2} P\left(t, \lambda_{\mathrm{c}}(t), \lambda\right) D_{\mathrm{s}}(\lambda) T(\lambda) \mathrm{d} \lambda,
$$




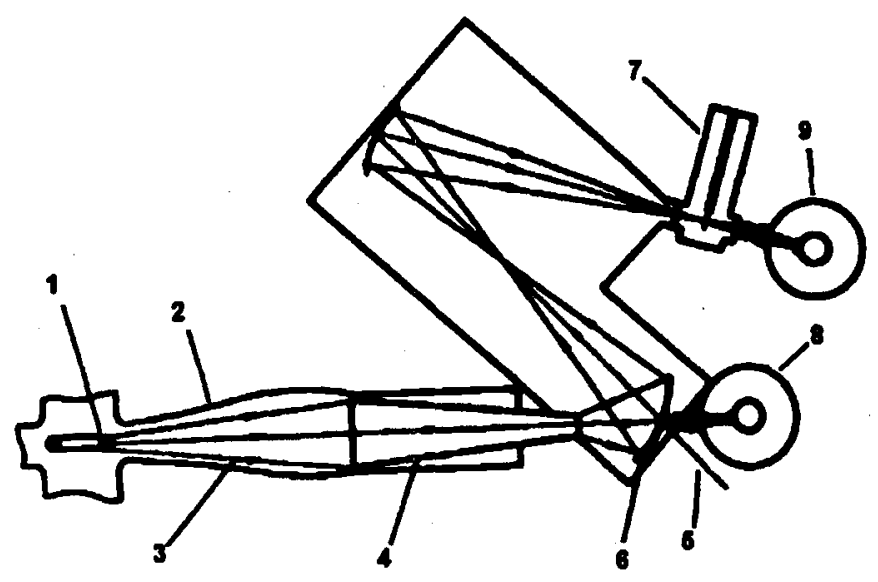

Fig. 1. Experimental setup and optical channel for HTSC films transmission spectra measurement: (1) electron ring, (2) vacuum chamber, (3) synchrotron radiation, (4) light cone ring, (5) laser beam, (6) spherical mirrors, (7) cryostat with sample, $(8,9)$ IR detectors.

where $D_{\mathrm{s}}(\lambda)$ is the spectral sensitivity of the detector and $P\left(t, \lambda_{\mathrm{c}}(t), \lambda\right)$ is the prompt SR spectrum of the electron ring.

In order to calculate the temporal evolution of SR-spectra it is necessary to determine $\lambda_{c}$ with a pinpoint accuracy. It was proposed in Ref. [3] to measure $\lambda_{c}$ with the help of the SR-spectrum slope in its short-wave region, where $\lambda \ll \lambda_{c}$. The slope magnitude is determined by the ratio of the SR power $P(\lambda)$ at different wavelengths $\lambda_{1}$ and $\lambda_{2}\left(\lambda_{1}>\lambda_{2}\right)$

$$
\lambda_{c}=\frac{\lambda_{1} \lambda_{2}}{\lambda_{1}-\lambda_{2}} \ln \left[\frac{P\left(\lambda_{1}\right)}{P\left(\lambda_{2}\right)}\left(\frac{\lambda_{1}}{\lambda_{2}}\right)^{5 / 2}\right] .
$$

Two detectors were used for measurements with the narrow band width filters:

$$
\begin{aligned}
& \lambda_{1}=12 \mu \mathrm{m}, \quad \Delta \lambda=2 \mu \mathrm{m}, \\
& \lambda_{2}=6 \mu \mathrm{m}, \quad \Delta \lambda=2 \mu \mathrm{m} .
\end{aligned}
$$

The detectors' calibration was done on the infrared monochromator, using the black body radiation.

The dependence $\lambda_{\max }=0.42 \lambda_{\mathrm{c}}$ was measured with respect to the electron ring compression time, obtained with simultaneous measurement of signal amplitudes from two detectors installed behind the optic channel. During a time of about $1 \mathrm{~ms}$ the wavelength of the maximum changes from $50 \mu \mathrm{m}\left(t_{0}=1.7 \mathrm{~ms}\right)$ to $20 \mu \mathrm{m}\left(t_{0}=2.5 \mathrm{~ms}\right)$.

To measure the transmission spectrum of a HTSC film, an optic channel was used, consisting of a conical light guide with a cryostat, where a YBCO film with a $\mathrm{MgO}$ substrate was placed. The cryostat temperature was adjusted in $20 \div 300 \mathrm{~K}$ range. 
Th IR-detector was manufactured with a silicon doped-B resistor and was arranged in the additional cryostat under the liquid helium temperature. oscilloscope.

The IR detector signal was transmitted through the preamplifier to a storage

The measurements were performed for the temperatures $T=100 \mathrm{~K}$ and $T=20 \mathrm{~K}$. The critical temperature of the superconducting transmission for YBCO film with a thickness of $800 \AA$ was $T_{\mathrm{c}}=80 \mathrm{~K}$.

The superconducting transition was controlled continuously by means of resistive signals from two contacts on the film. The YBCO was in a polycrystalline form.

A preliminary measurement demonstrated the absence of bolometric effects under irradiation of the pulsed SR-beam. The signal's ratios $\left(\mathrm{d} U_{\mathrm{S}} / \mathrm{d} t\right) /\left(\mathrm{d} U_{\mathrm{N}} / \mathrm{d} t\right)$ for radiation passed through the film under different temperatures are demonstrated in Fig. 2. S corresponds to the $T=20 \mathrm{~K}$ temperature and $\mathrm{N}$ corresponds to $T=100 \mathrm{~K}$.

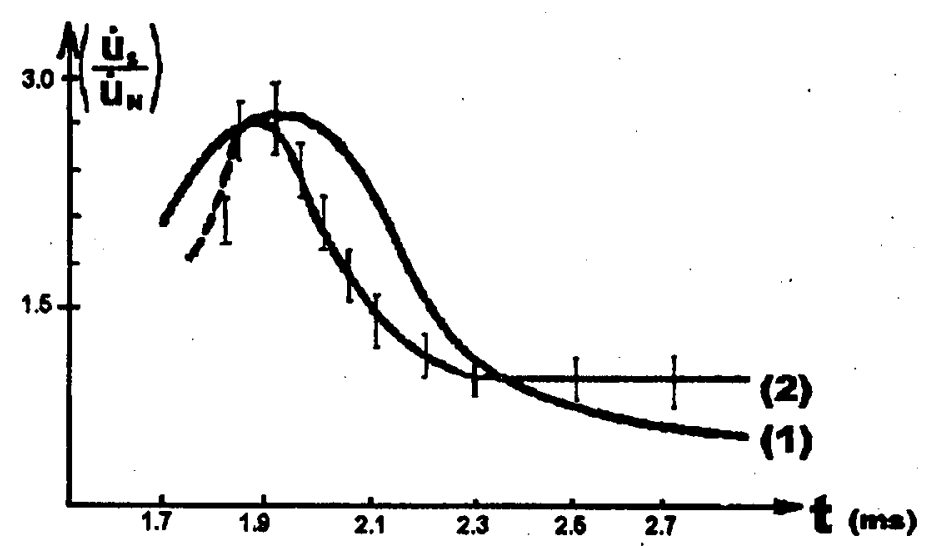

Fig. 2. Transmission using BCS theory, $2 \Delta=20 \mathrm{meV}$ - curve; experimental time dependence of the detector signal derivative $\left(\mathrm{d} U_{\mathrm{S}} / \mathrm{d} t\right) /\left(\mathrm{d} U_{\mathrm{N}} / \mathrm{d} t\right)-$ points $\times$. The detector measured SR passed through the YBCO film. Measurements were performed at two temperatures: $T=20 \mathrm{~K}(\mathrm{~S})$ and $T=100 \mathrm{~K}(\mathrm{~N})$.

As it may be seen, there is a significant effect in the change of YBCO film transmission. The closed curve to the experimental points is the one calculated for an energy gap of $2 \Delta=20 \mathrm{meV}$. It is rather difficult to observe the $2 \Delta$ dependence by means of oscillographic registration due to the low exactness of the amplitude's measurements.

The data qualitatively reflect the energy gap realization in the YBCO transmission spectra under $T=20 \mathrm{~K}$ and provides a magnitude for $2 \Delta: 2 \Delta=20 \mathrm{meV}$.

The accuracy variation of this magnitude by minimization of the expression in Eq. (2) to the experimental data, using the expression for the transmission coefficient provided by BCS theory, is about $30 \%$. This way, the obtained magnitude for the energy gap width is $2 \Delta=(20 \pm 6) \mathrm{meV}$. 


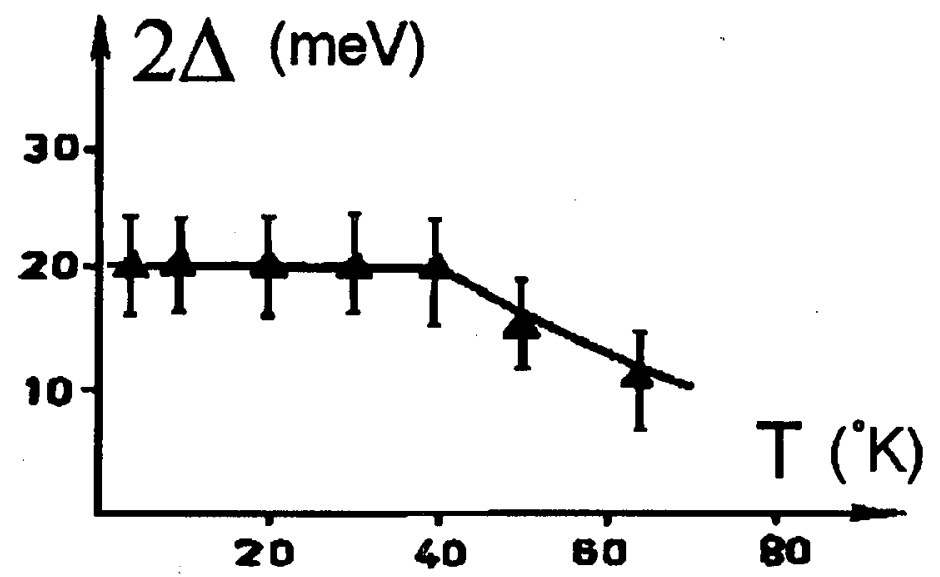

Fig. 3. Energy gap $(2 \Delta)$ vs. temperature.

During the experiments the energetic gap vs. temperature was also measured as shown in Fig. 3.

\section{References}

[1] W.D. Duncan, G.P. Williams, Appl. Opt. 22, 2914 (1983).

[2] T. Nanba, Rev. Sci. Instrum. 60, 1680 (1989).

[3] G.P. Williams, P. Takacs, R. Klaffky, H. Shleifer, Nucl. Instrum. Methods A 246, 165 (1986).

[4] S.I. Tyutyunnikov, V.N. Shaliapin, V.S. Shevtzov, A.S. Shenlin, JINR Commun. P14-91-144 (1991) (in Russian).

[5] G.V. Dolbilov, A.I. Maltzev, U.P. Sarantzev, A.P. Sumbaev, S.I. Tyutyunnikov, V.N. Shaliapin, Report of 10th Meeting on Charged Particle Accelerators, JINR Commun. D9-87-105, Dubna 1986, p. 390 (in Russian). 